\section{Immunglobuline leitliniengerecht anwenden}

_ Einigen Patienten mit Autoimmunkrankheiten, wie z.B Pemphigus vulgaris, kann mit einer konventionellen immunsuppressiven Therapie nicht geholfen werden. Intravenöse Immunglobuline (IVIG) sind dann oft der letzte Ausweg. Die vor kurzem erschienenen Leitlinien zur Anwendung von hochdosierten IVIG in der Dermatologie sollen dem behandelnden Hautarzt die Therapieentscheidung erleichtern [Enk A et al. Eur J Dermotol 2009; 19: 90-8]. „Das Ziel der Leitlinie war es, standardisierte Vorgehensweisen zu etablieren, um die Qualität der Therapie der Patienten zu verbessern und um evidenzbasierte Empfehlungen zu erstellen. Damit sollen aber auch die behandelnden Ärzte von der rechtlichen Seite her abgesichert und die Erstattung der Therapiekosten durch die Krankenkassen erleichtert werden“, erklärte Prof. Alexander Enk, Heidelberg.

Zunächst wurde festgelegt, welche Erkrankungen mit IVIG behandelt werden können: Dermatomyositis, blasenbildende Autoimmunerkrankungen (wie Pemphigus vulgaris, Pemphigus foliaceus und benignes Schleimhautpemphigoid), Vaskulitiden, systemischer Lupus erythematodes und toxische epidermale Nekrolyse (TEN).

Ein wichtiges Ergebnis, auf das sich die Experten einigten: Die Anwendung von

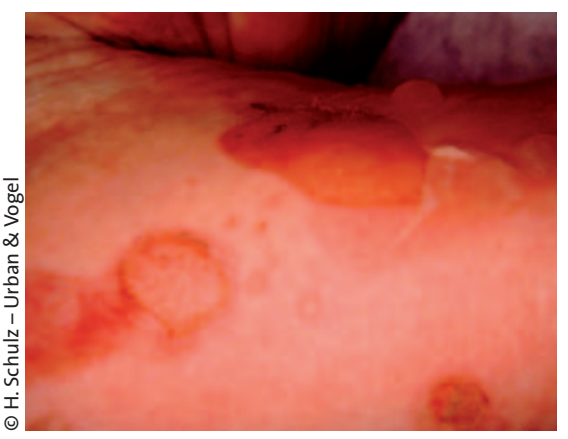

Intravenöse Immunglobuline sind beim Pemphigus vulgaris oft die letzte Therapieoption.
IVIG sollte niemals als First-line-Therapie erfolgen. Ausnahmen stellen die Dermatomyositis mit fulminantem Verlauf, schwerer Myolyse oder Paralyse, das Kawasaki-Syndrom sowie die TEN dar.

Die Experten legten außerdem eine Standarddosierung der IVIG von 2 g/kg KG als Bolus fest, mit Ausnahme der TEN, bei der die Dosis $3 \mathrm{~g} / \mathrm{kg}$ KG betragen sollte. Die Gesamtmenge sollte über zwei bis fünf Tage verteilt gegeben werden. Beim Kawasaki-Syndrom und bei der TEN ist nur ein IVIG-Therapiezyklus notwendig. Bei allen anderen Erkrankungen sollte IVIG initial alle vier Wochen, bei deutlicher klinischer Besserung und Stabilität alle sechs Wochen verabreicht werden. Sollte nach sechs Behandlungszyklen keine nennenswerte Besserung eingetreten sein, ist die IVIG-Therapie abzusetzen.

nz

Symposium „Intravenous immunoglobulins in dermatological autoimmune disorders“, 39. Annual ESDR Meeting, Budapest, 10.9.2009 (Veranstalter: Biotest).

\title{
SLIT mit monomerem Allergoid wirkt rasch und sicher
}

- Zur spezifischen sublingualen Immuntherapie (SLIT) bei Pollen- und Milbenallergien steht neben dem ,traditionellen“Verfahren nun mit Lais ${ }^{\circledR}$ auch in Deutschland eine Variante zur Verfügung, die immunogene Wirksamkeit mit sehr geringem Nebenwirkungspotenzial verbindet. Die Allergenextrakte in Lais ${ }^{\circledR}$ wurden nach einem patentierten Verfahren zu monomeren Allergoiden (Monoiden) carbamyliert, erklärte Prof. Giovanni Passsalacqua, Genua. Dadurch behält das Protein seine molekularen Konformation, aber seine IgE-Bindungsaffinität sinkt. Dieser Kunstgriff senkt die Allergenität-bedeutet also mehr Sicherheit bei voller immunologischer Effektivität.

Ein weiterer Vorteil des Monoids ist die höhere Bioverfügbarkeit dank besserer Magensaftresistenz. Eine wirksame Immuntherapie mit „vergleichbaren Ergebnissen wie bei anderen Sublingualtabletten" ist bei Einsatz des Monoids folglich mit relativ niedrigen Allergendosen möglich, betonte Prof. Ralph Mösges, Köln.

Das sublinguale Allergoid, das in Italien bereits seit über 15 Jahren auf dem Markt ist, gibt es in Tabletten- und Tropfenform zur
Hyposensibilisierung gegen Gräser-, Baum(Olive, Zypresse, Birke, Erle) und Kräuterpollen (Beifuß, Ragweed) sowie gegen Hausstaubmilben und Katzenallergene. Seine Effektivität bei allergischer Rhinitis und allergischem Asthma wurde in zahlreichen Studien unter Beweis gestellt; die Symptomatik und auch der Bedarf an antiallergischer Zusatzmedikamenten sanken signifikant. Selbst ein kurzfristiger Therapiestart vier Tage vor dem Pollenflug kann sich lohnen. Die saisonale Erhaltungstherapie umfasst zwei Monate, ebenso ist aber auch eine ganzjährige Therapie möglich. Kinder ab sechs Jahren können ebenso behandelt werden wie Erwachsene jeden Alters. ue

Symposium „Neue Erkenntnisse zur Pharmakokinetik und Wirksamkeit der spezifischen Immuntherapie mit Sublingualtabletten“, Berlin, 29.10.2009 (Veranstalter: Lofarma Deutschland).

\section{US-Zulassung für C1-Esterase-Inhibitor}

— Neuerdings können Patienten mit Hereditärem Angioödem (HAE) nun auch in den USA akute Attacken mit der Injektion von C1-Esterase-Inhibitor-Konzentrat kupieren - die US-Gesundheitsbehörde FDA hat Berinert ${ }^{\circledR}$ kürzlich als erste und bisher einzige Akuttherapie für die potenziell lebensbedrohliche Erkrankung zugelassen.
Zwar ist das Krankheitsbild sehr selten, doch da das klinische Erscheinungsbild häufig nur schwer zuzuordnen ist, wird es darüber hinaus oft lange verkannt. Die deutsche Internetseite www.hae-erkennen.de will deshalb für mehr Aufklärung sorgen.

Nach Informationen von CSL Behring 